\title{
Combined Bicycle and Google Street View Development of Virtual Street View system
}

\author{
Here Huan-Sheng Wang ${ }^{1}$, Wan-Shing Yang ${ }^{2 *}$, Li-Ming Hu${ }^{3}$, Yu-Ying Lin ${ }^{4}$, \\ Tsai-Hua Kang ${ }^{5}$, Wen-Kuei Hsieh ${ }^{6}$ \\ ${ }^{1}$ Dept. of Computer and Communication Engineering, National Kaohsiung University of Science and Technology \\ Kaohsiung City, Taiwan, R.O.C. \\ hswang@nkfust.edu.tw \\ ${ }^{2}$ Dept. of Computer and Communication Engineering, Hungkuo Delin University of Technology \\ New Taipei City, Taiwan, R.O.C. \\ yang@mail.hdut.edu.tw \\ ${ }^{3}$ Dept. of Computer Science, Paul Hsu Senior High School \\ Taoyuan City, Taiwan, R.O.C. \\ lmhu0405@gmail.com \\ ${ }^{4}$ Dept. of Business Administration, Hungkuo Delin University of Technology \\ Taipei, Taiwan, R.O.C. \\ roselin@ mail.hdut.edu.tw \\ ${ }^{5}$ Dept. of Computer Science and Information Engineering, Hungkuo Delin University of Technology \\ New Taipei City, Taiwan, R.O.C. \\ shakakang@yahoo.com.tw \\ ${ }^{6}$ Applied Foreign Languages Center, Hungkuo Delin University of Technology, \\ New Taipei City, Taiwan, R.O.C. \\ wkhsieh@mail.hdut.edu.tw \\ * Corresponding Author
}

\begin{abstract}
In this study, we try to combine the indoor bicycle riding with the Google Street View to simulate the outdoor bicycle riding to increase the pleasure of riding. Bicycle riding has been popular with many people and is susceptible to climate, limiting the riding time. Indoor bike riding has no such limitation, but monotonous indoor bike riding is boring. Google Street View is developed by Google and Google Street View can provide horizontal direction $360^{\circ}$ and vertical $180^{\circ}$ Street panorama, so users can view the chosen city address and the scenery on both sides. Combining indoor bike riding with Google Street View will make the indoor bike riding much more fun. In this study, the Arduino Uno microcontroller is used to detect the direction of the bike handle and the rotation speed of the bike tire and then sends these signals to Google Street View. In this way, we can achieve an interesting sports with virtual Street View.
\end{abstract}

Key words: Bicycle, Google Street View, Virtual Street View, MCU

\section{Introduction}

Bicycles are a very popular means of transportation for human beings and bicycles are also a popular sports event. However, outdoor bike riding is often limited by weather, unknown road conditions and time constraints. This study tries to combine the indoor bike riding with Google Maps street service to create an indoor riding simulation system. This simulation system can make people familiar with the route to be visited through this virtual reality system, and this simulation system can provide great help for self-travel route planning or leisure route planning. In addition, for the busy work and lack of sports of modern people, it is also possible to increase the fun of using indoor bicycles through this bicycle virtual reality system.

Google Street View has been used more and more in virtual reality, such as leisure development project [1], tree detection and species identification in a city [2] and roadside parking lot spacing study [3]. With this system, users can make a bike riding freely and users can go where they want to go, as long as the Google Street View exists.

\section{Background}

The module used in this study is introduced as follows:

\section{A. Microcontroller ATmega328P}

From the low cost microcontrollers to the high performance microcontrollers, there are lots of microcontrollers on the market. In consideration of our application, the ATmega328P [5] is used. This microcontroller is adopted in the Arduino UNO board, and the retail price is very competitive due to the huge market demand. This microcontroller is 8-bit AVR RISC-based microcontroller. And ATmega328P has 32KB ISP flash memory, 1024B EEPROM, 2KB SRAM, 23 general purpose I/O lines, 32 general purpose working registers, three flexible timer/counters with compare modes, internal and external interrupts, serial programmable USART, a byte-oriented 2-wire serial interface, SPI serial port, a 6-channel 10-bit A/D converter. By executing powerful instructions in a single clock cycle, the ATmega328P achieves throughputs approaching 1 MIPS per MHz, balancing power consumption and processing speed. As shown in TABLE I. List the characteristics of ATmega328P. 
TABLE I

ATmega328P FEATURES

\begin{tabular}{|l|l|}
\hline $\begin{array}{l}\text { High performance, low power } \\
\text { AVR } ® \text { 8-bit microcontroller }\end{array}$ & RISC architecture \\
\hline 131 instructions & $\begin{array}{l}\text { Maximum number of } \\
\text { I/O pins: } 23\end{array}$ \\
\hline UART : 1 & SPI : 2 \\
\hline Analog comparator: 1 & ADC: 8 channels, \\
& 10 -bit resolution \\
\hline EEPROM (KB) : 1 & Flash Memory: $32 \mathrm{~KB}$ \\
\hline Temperature range: $-40-+85^{\circ} \mathrm{C}$ & SRAM $(\mathrm{KB}): 2$ \\
\hline
\end{tabular}

\section{B. Reed Switches}

Reed switches [6] are magnetically-actuated electrical switches (not magically-actuated, though it seems that way sometimes). When the body of the switch is exposed to a magnetic field - like a magnet or even a strong electrical current - two ferrous materials inside pull together, the connection closes, and current can flow. In absence of a magnetic field, the switch opens. The characteristic of the reed switch used in our system is described in TABLE II.

TABLE II

CHARACTERISTIC OF REED SWTICH

\begin{tabular}{|l|l|}
\hline The total length is $20 \mathrm{~mm}$. & $\begin{array}{l}\text { Temperature: } \\
-10^{\circ} \mathrm{C} \sim+50^{\circ} \mathrm{C}\end{array}$ \\
\hline Contact arrangement: N.C. & Magnet: Nd FeB N35 \\
\hline $\begin{array}{l}\text { rated current: } 0.2 \mathrm{~A} / 50 \mathrm{VDC} \\
\text { (resistive load) }\end{array}$ & $\begin{array}{l}\text { Detection Distance: } \\
\text { MC-25 : 20mm min }\end{array}$ \\
\hline
\end{tabular}

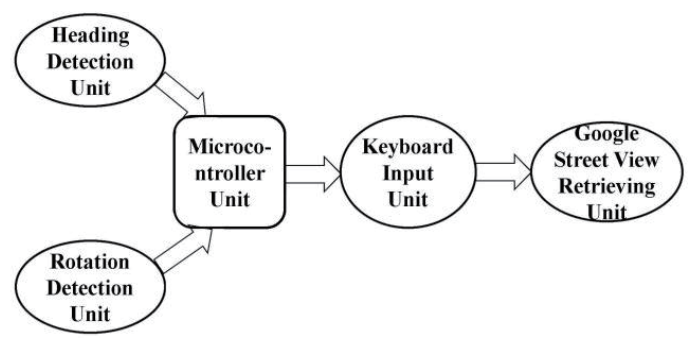

Fig. 1 System Diagram

\section{System Diagram}

The system diagram is shown in Fig. 1. There are heading detection unit, rotation detection unit, microcontroller unit, keyboard input unit and Google Street View retrieving unit. The movement of the rider is detected by the heading unit and the rotation unit. And signals are processed by the microcontroller. These processed signals imitate the keyboard signal and are sent to Google Street View via internet. Finally street maps are shown on the screen.

\section{A. Heading Detection Unit/Rotation Detection Unit}

The heading detection unit detects three heading directions: forward movement, left movement and the right movement.
These direction detections are achieved by two reed switches. One switch is put on the left handle and the other is put on the right handle. The rotation detection unit is detecting the rotation speed of the bike. So the system can decide the refresh rate of the Street Map.

\section{B. Microcontroller Unit}

The function of the microcontroller is to collect the bike heading signal and the rotation signal to calculate a new street position and remap these signals to the move forward key, turn left key or turn right key of the Google Street View system. In this system, the Arduino UNO ATmega328P is doing this remapping task.

\section{Keyboard Input Unit}

There are four input control keys for the Google Street View. There are left arrow key, right arrow, up arrow and down arrow. The ATmega328P is responsible for translating the heading signal and rotation signal into these four keys.

\section{System Flow Graph}

The system flow graph is shown in Fig. 2. The system checks three movement: forward movement, left movement, and right movement. If certain movement is detected, then the corresponding signal will be sent to the Google Street View.

By sending the left arrow key signal, the left side street view will be retrieved. And by sending the right arrow key

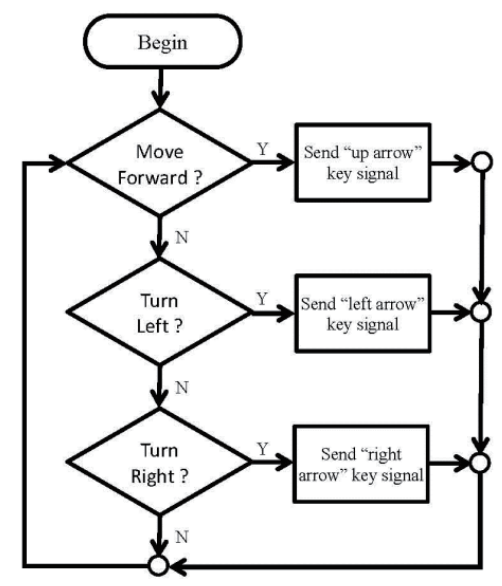

Fig. 2 System Flow Graph

signal, the right side street view will be retrieved.

\section{Technical Analysis}

The circumference of the bike tire in our system is 203.6CM. The refresh distance of Google Street View is 10 meter. In our system setting, when the pedal rotates 360 degrees, the bike moves forward 10 meter. So when the pedal rotates 360 degrees, the Street View refreshes once. Also when the bike handle turns 40 degrees from the center, the left turn signal or the right turn signal will be triggered. So the left side street view or the right side street view can be obtained. 


\section{Conclusion}

In this study, a simple indoor bike riding system is presented. This bike riding system combines indoor bike riding with Google Street View. And Arduino UNO is adopted as the system kernel. UNO collects the heading and the rotation signals and remaps these signal into movement signals. And the corresponding street scenery will be retrieved from the Google. This system is a combination of internet services, entertainment and sports. This system is fun and practical. And this system provides one more choice for indoor leisure activities.

\section{References}

[1] Yi-Wei Chen (2014), Using Virtual Reality to Provide the Street View of Leisure Time Opportunities for People, Dept. of Maritime information and Technology, National Kaohsiung University of Science and Technolog, Master's Thesis.

[2] SteveBranson, et al., From Google Maps to a fine-grained catalog of street trees, ISPRS Journal of Photogrammetry and Remote Sensing, Vol. 135, pp. 13-30, Jan. 2018.

[3] Paul Schimek, Bike lanes next to on-street parallel parking, Accident Analysis and Prevention, Vol. 120, pp. 74-82, Nov. 2018.

[4] Li-Ming Hu (2018), Research on the Combination of Bicycle and Street View Interactive Production, Dept. of Digital Technology Design-Master Program in Toy and Game Design College of Science, National Taipei University of Education, Master's Thesis.

[5] Arduino Home, Retrieved from https://www.arduino.cc/ (Oct. 01, 2018).

[6] Shincluster Electronics Co., Ltd., Retrieved from http://www.ecluster.com.tw/shincluster/index.php/tw/ (Oct. 01, 2018) 
Recent Developments on Information and

Communication Technology (ICT) Engineering- Meen, Yang \& Zhao ISBN: 978-981-14-2136-5 